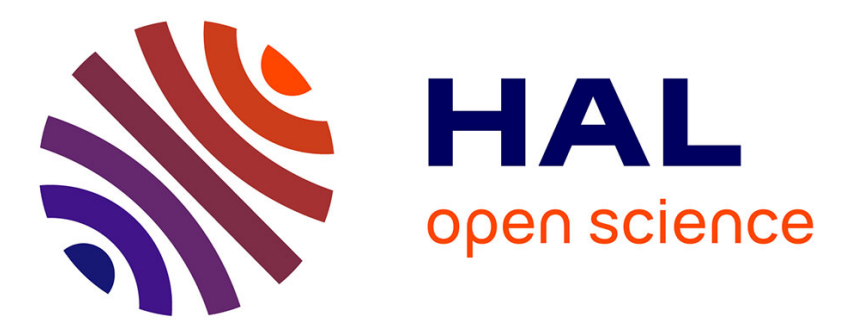

\title{
Air pollution and psychiatric disorders: Current and future challenges
}

\author{
Baptiste Pignon, Andrei Szöke, Gilles Forêt, Antoine Pelissolo, Marion \\ Leboyer, Franck Schürhoff
}

\section{- To cite this version:}

Baptiste Pignon, Andrei Szöke, Gilles Forêt, Antoine Pelissolo, Marion Leboyer, et al.. Air pollution and psychiatric disorders: Current and future challenges. Bipolar Disorders, 2020, 22 (5), pp.437-439. 10.1111/bdi.12961 . inserm-03138047

\section{HAL Id: inserm-03138047 https://www.hal.inserm.fr/inserm-03138047}

Submitted on 10 Feb 2021

HAL is a multi-disciplinary open access archive for the deposit and dissemination of scientific research documents, whether they are published or not. The documents may come from teaching and research institutions in France or abroad, or from public or private research centers.
L'archive ouverte pluridisciplinaire $\mathbf{H A L}$, est destinée au dépôt et à la diffusion de documents scientifiques de niveau recherche, publiés ou non, émanant des établissements d'enseignement et de recherche français ou étrangers, des laboratoires publics ou privés. 


\section{Air pollution and psychiatric disorders: current and future challenges}

Baptiste Pignon $^{1}$, Andrei Szöke ${ }^{1}$, Gilles Forêt ${ }^{2}$, Antoine Pelissolo ${ }^{1}$, Marion Leboyer ${ }^{1}$, Franck Schürhoff ${ }^{1}$

${ }^{1}$ AP-HP, DMU IMPACT, Hôpitaux universitaires Henri-Mondor, Pôle de Psychiatrie, Créteil, 94000, France ; Inserm, U955, team 15, Créteil, 94000, France ; Fondation FondaMental, Créteil, 94000, France ; UPEC, Université Paris-Est, Faculté de médecine, Créteil, 94000, France.

${ }^{2}$ Laboratoire Interuniversitaire des Systèmes Atmosphériques, CNRS/INSU UMR7583, Université Paris-Est Créteil et Université Paris Diderot, Institut Pierre Simon Laplace, Créteil, 94000, France. 
The recent article of Khan et al. ${ }^{1}$ opens a new field of research in bipolar disorders and other psychiatric disorders, which can give clues for a better understanding of their pathophysiology. These authors provide an analysis of two large national datasets, highlighting the issue of air pollution as a risk factor for major psychiatric disorders. The authors show the influence of different environmental factors, including air pollution characteristics, on the general population prevalence of four psychiatric disorders (bipolar disorder, major depression, schizophrenia, and personality disorders taken as a whole) in Denmark and the USA. Their results show that poor air quality is associated with an elevated prevalence of bipolar disorders both in US and Denmark, and with elevated prevalence of schizophrenia, major depression and personality disorders also being associated with poor air quality in Denmark.

The Danish study may be the more interesting of the two studies. This study analyzes data pertaining to the influence of childhood-exposure (first 10 years of life) to global air pollution (based on 14 air quality indicators) in more than 1,400,000 subjects, born between 1979 and 2002, and followed-up until 2016 (ages 14 to 37). The exposure was measured at an individual level, based on each individual residential address. The analyzes were adjusted for current urbanicity as well as main sociodemographic factors. Another strength of this study is the utilization of the national Danish health outcome registry, thereby limiting the effects of missing data. The results show that the rates of all noted disorders are elevated with increasing levels of air pollution exposure. Air pollution is shown to differentially interact with psychiatric diagnoses, with for instance in the group having the highest vs. lowest childhood exposure to air pollution: $+29 \%$ for bipolar disorders, $+50 \%$ for major depression, $+148 \%$ for schizophrenia, and $+162 \%$ for personality disorders. The completeness of the data is strengthened by the fact that exposure measured predates diagnosis, especially as this pertains to a critical neurodevelopmental period. Overall, such data provides strong support for a causal relationship between air pollution and psychiatric disorders.

The US study provides an opportunity to see if these finding are replicated ${ }^{2}$. However, the two studies are quite different in their general design, data available and statistical analyses used. The data in the USA study only provides data on air pollution exposure at a county (i.e., ecological - not individual) level, and therefore is weaker given that it is subject to the "ecological fallacy", whereby associations at individual level may not parallel those observed at group (ecological) level. The timing of the measures is also significantly different 
in the USA, with psychiatric prevalences in each country being measured between 2002-2013, and air pollution levels being used between the years 2000 to 2005. Consequently, the USA exposure to air pollution and diagnostic data overlap, and contrast to the clear neurodevelopmental perspective that can be drawn from the Danish data. This is problematic as etiological factors can predate psychiatric diagnoses by decades, and the level of air pollution has probably changed in the interval, which is clearly shown in the Danish data where a decrease occurred between 1980 and 2000. Furthermore, county and international migration are frequent in the USA, and may have biased the analyses, e.g., people currently resident in a given county may have been exposed to air pollution in another county at a crucial developmental time-point.

Although the USA study has these limitations, it does also have a number of strengths, including: quality of data on pollution (based on 87 air pollutants concentrations); more precise detailing of other potential risk factors (e.g., counties' water and land pollutions, quality of built environment, population density, weather characteristics, level of urbanicity, ethnicity and several other socioeconomic factors); and a rigorous level of statistical methods (including correction for multiple testing and correction for spatial autocorrelation). Moreover, the authors used several alternative procedures to detect cases (e.g., for bipolar disorders, based solely on assurance claims, or additionally on lithium prescriptions), without significant changes in their results.

However, by contrast with the Danish study, the clinical data of the USA study have a number of limitations, including quantitative (e.g. for schizophrenia: $49.2 \%$ of the data are missing) and possibly qualitative (the most frequent psychiatric diagnosis in the 0-5 age-band are personality disorders, which are disorders that typically emerge in adolescence or early adulthood). Consequently, the associations reported in the USA study may be biased ${ }^{3}$. The only positive association between neuropsychiatric disorders and air pollution in the US sample after testing for the same disorders (plus Parkinson disease and epilepsy) is for bipolar disorders, which showed an increased prevalence of more than $25 \%$ between the lowest and the highest exposed counties. However, given the methodological and clinical case data differences, inconsistent results were to be expected.

The Khan et al. study is the first to analyze the influence of air pollution on the risk of bipolar and personality disorders. In comparison to other environmental risk factors, 
particularly psychosocial, the influence of air pollution has been relatively little investigated psychiatric disorders. Several recent studies indicate significant associations of air pollution with schizophrenia, depression and autism spectrum disorders (ASD) ${ }^{4}$. In the Khan et al. article, the epidemiological analyses are very rich and, beyond analyzes of the air pollution, with some important findings. For example, urbanicity is one of most consistent factors associated with psychiatric disorders, and is considered as a marker of different risk factors. Exposure to air pollution has been suggested as an explanation for this link. However, the two studies (Danish and USA) both found significant associations with urbanicity (for bipolar disorders in US, and for the four considered psychiatric disorders in Denmark) after adjustment for air pollution. Thus, air pollution may partly, but not fully, explain the link between psychiatric disorders and urbanicity. Moreover, ethnicity was the best predictor of the USA prevalence for mood disorders (bipolar disorders and major depressive disorder), as well as schizophrenia.

As noted, the epidemiological evidence linking air pollution and psychiatric disorders is scarce, which is surprising given the biological plausibility of this association. Air pollutants increase brain inflammatory and cytotoxic damage, including via microglia activation, with resultant oxidative damage and neuronal loss ${ }^{4}$. Air pollutants may also act via hormonal dysregulation, including by dysregulating the hypothalamic-pituitary axis. Such air pollutant-driven phenomena are involved in both the etiology and severity of psychiatric disorders. Animal preclinical data indicates at least three pathways whereby air pollutants modulate brain function: indirect transport by systemic inflammation, and direct transport by way of intravascular brain macrophages or via nasal respiratory damage. Given the paucity of studies on air pollution and psychiatric conditions, there are no confirmation of a link between air pollution and a given psychiatric disorder. To date, the strongest association of air pollution and neuropsychiatric conditions is with ASD, especially from exposure in pregnancy and early life. Clearly, the association of air pollution with bipolar disorders in these two samples is of some importance, but requires further investigation.

Overall, despite several limitations and the need for replication, this article has a number of merits, including providing novel data with clear paths for future research, as well as highlighting methodological issues. Clearly, the effects of air pollution on psychiatric disorder risk will be generate diverse future research, including as to how it interacts with other psychiatric risk factors, such as psychosocial risk factors. Such interaction would seem 
more the rule than the exception. Exposure-wide systematic approaches and multilevel logistic regression models with individual risk factors nested within contextual factors - thus, big data analyses - will be required to separate confounding and genuine phenomena. The exposure to air pollutants' influence on the pathoetiology and pathophysiology will require investigation from the prenatal period onwards until the onset of psychiatric disorders. It will also be important to identify the type of air pollutants driving these pathophysiological changes and as to whether there are any interactions across air pollutants. Recent data has highlighted the detrimental effects of fine and ultra-fine particles in a wide array of disorders, including lung, cardiovascular, cerebral, and infectious disorders 5 .

Clearly, it will be important to determine any crucial, developmental temporal windows (including in-utero) for each air pollutant and for each psychiatric disorder. It is also important to note that the air pollution studies on psychiatric disorders to date focused exclusively on outdoor air pollution. The role of indoor pollution for most individuals may be at least as important, including any interactions with outdoor pollutants. Large and specific longitudinal studies are necessary. The investigation of brain damage and the biological pathways linking air pollution and psychiatric pathophysiology will require preclinical investigations, including as to the consequences of acute and/or chronic exposure to varying levels of pollutants at different developmental time-points. Such data should better clarify the pathoetiology and pathophysiology of psychiatric disorders, as well as provide significant preventative targets. 


\section{REFERENCES}

1. Khan A, Plana-Ripoll O, Antonsen S, et al. Environmental pollution is associated with increased risk of psychiatric disorders in the US and Denmark. PLOS Biology. 2019;17(8):e3000353.

2. Moonesinghe R, Khoury MJ, Janssens ACJW. Most Published Research Findings Are False-But a Little Replication Goes a Long Way. PLOS Medicine. 2007;4(2):e28.

3. Ioannidis JPA. Air pollution as cause of mental disease: Appraisal of the evidence. PLOS Biology. 2019;17(8):e3000370.

4. Buoli M, Grassi S, Caldiroli A, et al. Is there a link between air pollution and mental disorders? Environ Int. 2018;118:154-168.

5. Pope C. Arden, Lefler Jacob S., Ezzati Majid, et al. Mortality Risk and Fine Particulate Air Pollution in a Large, Representative Cohort of U.S. Adults. Environmental Health Perspectives. 2019;127(7):077007. 